$x-602-74-45$

PREMUNT

IUSA 话 X- 70592

\title{
ERROR ENHANCEMENT \\ IN GEOMAGNETIC MODELS \\ DERIVED FROM SCALAR DATA
}

(H. 1-TY-X-76592) EETOF ENHANCEMENT IY

GF - GREIIC MOLEIS DERIVED FECA SCALAK

DA (NASA) $25 \mathrm{p} \mathrm{HC} \$ 4.25$ CSCI $20 \mathrm{~J}$

$\$ 74-17117$

03,13 Jucias

\author{
DAVID P. STERN \\ JOSEPH H. BREDEKAMP
}

G SIC - GCODARD SPACE FLIGHT GENTER

GREENBELT, MARYLANO 


$$
\begin{aligned}
& \text { Error Enhancerent in Geomabnetic } \\
& \text { Models Derised from scalar Data }
\end{aligned}
$$

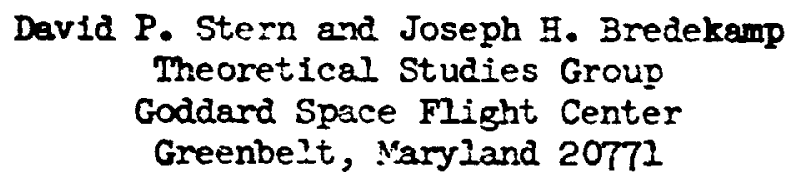

$$
\text { A b } s \text { t } \mathbf{r} \text { a }
$$

Hodels of the main geomagnetic fleld are generally represented by a scalar potential $\gamma$ expanded in a finite number of spherical harmonics. In the last decade, such models heve been derived mainly by a recursive iteration method from the fleld. magnitude $F$ observed by satellites in low-altitude polar orbits. Very accurate observations of $F$ were used, but indications exist that the accuracy of models derived from them is considtiabls lower. Ont problem is that $F$ does not always characterize $\gamma$ uniquely: Backus has derived a class of counterexamples in which two different choices of $\gamma$ correspond to the same $F$. It is not clear vhether such ambiguity can be encountered in derieing $\gamma$ from $F$ in geomagnetic surveys, but there exists a connection, due to the fact that the counterexamples of Beckus are related to the dipole fleld, while the geomagnetic fleld is acmlnated bj its alpole component. If the models are recovered with a finite error (1.e. they cannot completely fit the data and consequently have a small apurious component) this connection allows the error in certain sequences of harmonic terms in $\gamma$ to be enhanced without unduly large effects on the fit of $F$ to the model. Computer almulations have demonstrated this effect, producing as a result models which fit the data of $F$ quite closely but yleld much poorer Its to the alrection of the magnet 1c vector. Possible remedies are discust 2 . An append $1 x$ also discusses a particlilar cless of flelds related to the counterexamples of Backus - for which it can happen that the recursive iteration deriving $\gamma$ from $F$ does not converge to the correct solution. 
INTRODUCTION

In order to derive a general vector field in scace, three incepencert scalars must be observed at each point - e.E. 3 orthogonal componunts, or the rield's magnitude and two anglec defining its direction. For the main geomagnetic field B - i.e. the part which originates dee inside the earth - such an observation is redunciant, since that field is completely derined by a scaler potential $y$

$$
\underline{B}=-\nabla \gamma
$$

The information needed to derive $\underline{B}$ nay thus be given by a single scalar function of position. In fact, there exists the additional constraint that $\gamma$ must be harmonic; this may be viewed as reducing the "information content" or $\gamma$ to that or a two-dimensional scalar furction, since only 2 indices appear in the spherical harmonic expansion of $\gamma$, traditionally written as

$$
\gamma=a \sum_{n=1} \sum_{m=0}^{m=n}(\theta / r)^{n+1} F_{n}^{m}(\theta)\left\{g_{n}^{m} \cos m \varphi+h_{n}^{m} \sin m \varphi\right\}
$$

( $a$ is the earth's radius). In satellite surveys it is relatively easy to massure accurately one of the three scalars associated with the fisld, namely the magnitude $|\underline{B}|$, comonly denoted in geomagnetic literature by the capital letter $F$. Accurate observation of $F$ requires neither information on the attitude of the observing spacecraft, not observation of the flexing of the boom carrying the magnetometer; such information is required for accurate determination of the fleld's direction, which consequently is much harder to perform.

Furthermore, satellites conducting surveys of the field generally do not valy their altitude by great amounts, so that their observations of $F$ tend to be distributed more or less over a two-dimensional surface - that of a sphere concentric with the earth. The preceding quelitative arguments do however suggest that the amount of information thus gathered may suffice to determine the rield. 
This has led to attempts to trade one scalar for the other, i.e. to derive $\gamma$ from observations of $F$ (of course, observea data must first be "cleaned" of fields of external origin, bu', this important point is not considered here). Zmuda [1958] appears to have been the first to propose this approach and its first major use occured in the analysis of Vanguard 3 data by Cain et al. [1062] - The derivation of ariels of the main field from satellite data has generaily been based on $F$

[ Heppner, 1963; Cain et al., 1967 ; Cain, 1971 ], in particular the models derived from observaticn: of $0_{0} 2,4$ and 6 [Cain and Sweeney, 1970] - The method used in slich work is described further on . Beceuse $F$ is easily observed down to intensities of $1 \gamma\left(=10^{-5}\right.$ gauss $)$ this has become the method of choice for mapping the geomagnetic field and models which ift observed $F$ within an r.m.s. deviation of $4-7 \gamma$ have been derived by it.

However, two problems arose - one experimental, the other theoretical. Experimentally, the few observations in which the vector direction of the fleld could be compared to the model indicated considerable deviations [Cohen, 1972; Woodman, 1272] - Alan, the results of airborne surveys Indicate (J. Cain, private comunication) that field components often deviate by $100-200 \gamma$ from the values prescribed by models.

The theoretical difficulty was pointed out by G. Backus [1968, 1970] who investigated the question of whether $F$ observed on a sphere uniquily defines the scalar potential $\gamma$ from which it car be derived. If the expansion (2) contains a finite number of terms it may be shown [Backus, 2968 ] that $F$ and $\gamma$ are uniquely related. However, if an infinite number of terms is allowed, that assertion turns out to be false and Backus [1970] furmished a class of counterexamples in which two quite distinct infinite expansions, similar tc (2), yleld identical forms of $F$ on the unit sphere $r=1$.

This alscovery has cast some doubt on the assertion that the gechasnetic scalar potential $\gamma$ is uniquely derivable from its corresponding $F$. So far, this question has not been resolved. 
What the present work shows is that the experimental discripancies between vector field observations $2: 2$ the precictions of the node: may have a mathematical origin, related to the $i \dot{*}$ or Backus. Specificaliy, It $w 1$ l be shown that when periect recovery $; \gamma$ is not possible due to Iinite observational erre $s$ of varic is origins - and erch of the cofficients of (2) contains a certain error, then this error is enhanced for certain sequences of terms related to those derived by Backus. The basic reason for the connection is that the counterexarales constructed by Backus are related to the dipole field, while the geometic field is known to be dominated by its dipole component. Computer simulations of the effect and possible remedies will also be described.

\section{TLE COUNTEREXAMPIE OF BACKUS}

Backus [1970] sought to determine whether it was possible, in three dimensional space, to find two harmonic functions $\phi_{2}$ and $\phi_{2}$, vanishing at infinity and differing by more than their algebraic signs, such that on the unit sphere $r=1$

$$
\left(\nabla \phi_{1}\right)^{2}=\left(\nabla \phi_{2}\right)^{2}
$$

He found it conventent to reformulate the problem in terms of the sum $u$ and the difference $v$ of these functions, which on $r=1$ have to satisfy

$$
\nabla u \cdot \nabla v=0
$$

Choosing $u$ to be the aipole potential

$$
u=\gamma_{d}=\cos \theta / x^{2}
$$

Backus assumed $v$ to be an infinite series of the rorm (2) and he then exanined the coefficients to see whether they could be made to 
satisfy (4). Not only did this turn out to be possible but a whole class of such solutions was found to exist. The general form of these solutions is best expressed if cne interchances the summations of equation (2) and writes

$$
\begin{aligned}
v= & \sum_{m=1}\left\{a_{m} \cos m \varphi \sum_{n=m}^{\infty} G_{n}^{m}(a / r)^{n+1} F_{n}^{m}(\theta)+\right. \\
& \left.+b_{m} \sin m \varphi \sum_{n=m}^{\infty} H_{n}^{m}(a / r)^{n+1} P_{n}^{m}(\theta)\right\}
\end{aligned}
$$

where for sake of definiteness the leading term in each of the summations over $n$ is set equal to unity

$$
G_{m}^{m}=\frac{H_{m}^{m}}{m}=1
$$

Then, provided the series in the in ler summations are suitably chosen (there exist two such sumations for each value of $m$, and they are actually the same - 1.e. $H_{n}^{m}=G_{n}^{m}$ ), equation (4) is satisfiec for any arbitrary choice of the coefficients $a_{m}$ and $b_{m}$. The series over $n$ are derived by recursion from their leading terms, and since these terms are fixed by (7), the series are unique. They are described in more detail in Appendix I and contain nonzero terms only for values of n and $m$ which sum up to an even number.

Strictly speaking, (6) satisfies (4) only if an infinite number of terms is used. We shall denote this limit of $\mathrm{v}$ by a superscript $\infty$ and write

$$
\nabla \gamma_{d} \cdot \nabla v^{\infty}=0
$$

If the number of terms in $v$ is finite, (8) no longer holds; however, the scalar product contributed by such a truncated $v$ (denoted by a superscript $f$ for "finite") may be small if $v^{\infty}$ is approached 
sufficiently closely. This may be yn a-ialiy writton as

$$
\nabla \gamma_{\mathrm{d}} \cdot \nabla \mathrm{v}^{f}=0(\varepsilon)
$$

where $\varepsilon \ll 1$ is a small parameter. In what follows we indicate for orevity all small quantities by $\varepsilon$; at. Least 3 distinct quantities of this sort appear, but devting to 3 ih of them a soarate notation merely encumbers the expressions and yiclds no new results. The magnitude of the right hand side or (9) depends on the exact form of $\mathrm{v}^{\vec{I}}$ - in particular, on the number $k$ of terms in the shortest summations over $n$ which appear in it. If $k$ is suficiently large, however, the expression (9) may be made arbitrarily small.

MODEI DERIVATION FROM SCAIAR DATA

In the derivations of models of the main geomagnetic field from a set of observations of $F=|\nabla \gamma|$, the value of $\gamma$ is determined by iterated linearization, starting from an initial approximation $\gamma_{0}$ of the scalar potent1al. Let $E \gamma_{2}$ be the correction to be added to $\gamma_{0}$ in order to obtain the true potential $\gamma$

$$
\gamma=\gamma_{0}+\varepsilon^{\gamma}
$$

Then

$$
\varepsilon \nabla \gamma_{2} \cdot \nabla \gamma_{0}=\frac{1}{2}\left\{F^{2}-\left(\nabla \gamma_{0}\right)^{2}\right\}-\frac{1}{2} \varepsilon^{2}\left(\nabla \gamma_{2}\right)^{2}
$$

If the term of order $\varepsilon^{2}$ is neglected and $\gamma_{1}$ is expanded in a finite series of form (2) with $N$ unknown coefficients, then (11) represents one linear equation with $\mathbb{N}$ unknowns for each point at which an obserration of $F$ was made.

Typically the number $M$ of such observations greatly exceeds $N$. Ir, for Instance, the ceries (2) for $\gamma_{2}$ is truncated past $n=n_{\max }$, then 


$$
N=\left(n_{\max }+1\right)^{2}-1
$$

Typically, $n_{\max }=10, N=120$, while the number of equations (one for each observation of $F$ ) may be of the order of 10,000. The bestfittine set of coefricients for $\gamma_{2}$ is then derived by least squares fitting, after which one replaces $\gamma_{0}$ by. $\left(\gamma_{0}+\varepsilon \gamma_{2}\right)$ and repeats the process, seeking a further correstion term $\gamma_{i}^{\prime}$. This procedure is continued until the r.m.s. difference hetween observed values of $F$ and those obtained from the model no longer shows any improvement. Various refinements may be included, e.g. dividing each of the equations (11) by $\left|\nabla \gamma_{0}\right|$ in order to avoid giving greater weight to observations in polar regions, where $F$ is larger.

Cases are known in which this procedure does not lead to the correct solution (Appendix II), but in analysis of simulated data from models representing the geomagnetic ilela this method tends to converge rapidly - to the "correct" solution, if the derived series is capable of expressing it, or to a small range of innal parameters corresponding to a "best-fitting series" if the data contain unresolved higher harmo.ilcs or other sources of small error.

ERROR EN HA NCE M N T

The basic equations used in the iteration are

$$
\varepsilon \nabla \gamma_{0} \cdot \nabla \gamma_{1}=\frac{1}{2}\left\{F^{2}-\left(\nabla \gamma_{0}\right)^{2}\right\}
$$

with one such equation being contributed by each observation point. These equations will lack a unique solution if the set of equations

$$
\nabla \gamma_{0} \cdot \nabla \gamma_{1}=0
$$

evaluated for the same points, does have a solution. In that case, given one solution of (13), new solutions may be obtuined by adding to it arbitsily multiples of solutions of (14). 
If all observations were conductad at the ame radial $2=0 \pm=2.9$, if $\gamma_{0}$ were the dipole ficld and if $\gamma_{1}$ contained in infinite number of terms, then hy (8) there would have existed a sulution to (124) and (13) would not have derined $\gamma_{1}$ uniqur $\therefore$ "None $=1$ these concitions

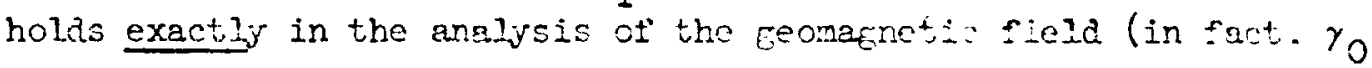
changes from one iteration to the next), tut they are all approximatriy valid and this, in the presence of finite erre may cause paobams.

In this section the finite spread in the racial distance $r$ will be neglected (its efiects are considera separately later or'. I. $^{\prime} \gamma_{0}$ now be separated into a dipole part and a non-dipole pait

$$
\gamma_{0}=\gamma_{\mathrm{d}}+\varepsilon \gamma_{\text {nd }}
$$

where $\varepsilon \ll 1$ is yet another small quantity.

If an exact solution for equations (13) does not, exist - due to truncation, experimentel error, unresolved contributions of external field sources etc. - then the "best fitting" result for $\gamma_{2}$ will consist of the sum of the "true solution" $\gamma_{t}$ and an "error" $\gamma_{\epsilon}$, each uf which will be expressed by a Iinite series similar to the one in equation (2). One can then write

$$
\varepsilon\left(\nabla \gamma_{\mathrm{d}}+\nabla \gamma_{\mathrm{nd}}\right) \cdot\left(\nabla \gamma_{t}+\nabla \gamma_{\mathrm{e}}\right) \cong \frac{1}{2}\left\{F^{2}-\left(\nabla \gamma_{0}\right)^{2}\right\}
$$

where $\cong$ will signify "best fitting by the least squares metrod" (for our particular set of observations).

Now a finite serles $\gamma_{\mathrm{e}}$ of form $(2 ;$ can in turn be resolved into two parts: a sum $\gamma_{\mathrm{eb}}$ of "Backus terms" simslar to those in (6), plus \& sum $\gamma_{\text {ei }}$ of independent harmonics. To perfor. such a separation one first isolates a.ll terms with $n=m$ and includes them in $\gamma_{\mathrm{eb}}$, while 
the remaining terms are assigned to $\gamma_{\mathrm{ej}}$. Each of the terms in $\gamma_{\mathrm{eb}}$ is now viewed as the leading cerm of a Backus-type series of the type isted in squation (6) a:" in Appendix I (including the coefficient $a_{m}$ or $b_{m}$ multiplying such a series), and appropriate higher-order terms are added to it to form the rest of its series, up to the highest $n$ and $m$ allowed by the Iinite expansion $\mathrm{o} z \mathrm{\gamma}_{\mathrm{eb}}$; co maintain the balance, the same terms that are added to $\gamma_{\mathrm{eb}}$ are subtracted from $\gamma_{\mathrm{ei}}$. Finally, those expansions in $\gamma_{\mathrm{eb}}$ deened to have (after truncation) too few terms $I$ (r) (9) to be valid (with the right hend side small enough) are transferrea from $\gamma_{\mathrm{eb}}$ to $\gamma_{\mathrm{ei}}$. This will certainly include all. those Backus series which contain only one term - and perhaps also those containing two. Equation (16) now may be written

$E\left(\nabla \gamma_{d}+\nabla \gamma_{n d}\right) \cdot\left(\nabla \gamma_{t}+\nabla \gamma_{e b}+\nabla \gamma_{e 1}\right) \cong \frac{1}{2}\left\{F^{2}-\left(\nabla \gamma_{0}\right)^{2}\right\}$

The cont ributions of the error terms to the left hand side are mainly due to scaiar products with the alpole term, which overshadows the non-cipolc term. If all coefficients involved in $\gamma_{\mathrm{eb}}$ and $\gamma_{\mathrm{ei}}$ were equal, then the contributicn to the scalar product associated with a cueiricient of $\gamma_{\mathrm{eb}}$ will on the average be one order in $\boldsymbol{\varepsilon}$ smaller thas the contribusion associated with one of the c'efficlents of $\gamma_{e i}$, since or equaition ( 9 ) the former conti bution gairs an extra order in $\mathcal{E}$.

Alternatively, if the least squarrs optimization adjusts all sources of error so that they contribute approximately equally, then the coefficients of $\gamma_{\mathrm{eb}}$ will be larger by a factor of the order of $\varepsilon^{-1}$ than those of $\gamma_{\mathrm{ej}}$, leading to an enhanced error in these terms.

A differont view of the same effect is gainod by writing the basic relation prior to linearization

$$
\left\{\nabla\left[\gamma_{a}+E\left(\gamma_{n d}+\gamma_{t}+\gamma_{e b}+\gamma_{e 1}\right)\right]\right\}^{2} \cong F^{\hat{x}}
$$


When the left hand side is expanded in orders of $\varepsilon$, the $\varepsilon^{0}$ term is simply $\left(\nabla \gamma_{d}\right)^{2}$ and all $O(\varepsilon)$ terms of the scelar potential contr1bute to the term or order $\varepsilon$, except fow $\gamma_{\mathrm{eb}}$, ihich due to (9) only contributes at the $\varepsilon^{2}$ level. Thus $T^{2} i_{i}$ inkmant.jv less sencitive to Iluctuations of $\gamma_{\mathrm{eb}}$, allowing its errors to be relatively lares without excessive efiects on the fit of $F$. Intuitivelv, the Backu. series may be viewed as modes at which a near-dipole field, derived : $n$ the manner described here, prefers to fluctuate - just as a bridge or a beam tend to oscillate in rertain modes related to their geometrical properties.

$S I M U I A T I O N$

i test of the preceding argument was performed as follows. A 120-term exparsion of $\gamma\left(r_{\max }=\pi c\right.$ was used to devive the corresponding values of $F$ c.t $144 \mathrm{C}$ points on the unit sphere. A program enalvzing these simulated data by the previously described method was then applied, but the derived models contained on 763,80 or 99 terms $\left(n_{\max }=7,8\right.$ or 9$)$. Up to 10 itcrations were performed, although the model generally showed little variation entar the second iteration; the resuits cited here therefore reier to the rit after 2 iterations. As a check a differer.t input ior $r$ was used and similar effects vere observed, al.though they are not included in the resilts listed here.

From the models, the predicted intensity $F_{m}$ was derived for the 1440 input point and at each point the deviaition

$$
\delta F=|F-F|
$$

was computed. The quantity $\delta F / F$ was then derived, its average was also found and representative table of its values at every 3 rd point was printed out, from which the worst (1.e. largest) value was picked visualiy. In addition, the mean angle $\delta \lambda$ (in radians) between the model field and the true field was wived: It is of the same order as the relative discrepancy $\left[B_{i} / B_{i}\right.$ in one of the components of $B$. As before, the worst case was aiso pinked out visually from a representative table. 
The results are given in Table 2 . The relativa accuracy of the direction is more than 10 tims worse than that on the megnitude, and the wors, fit in direction has an error atout 10 times larger st111. This is an eriect slightly larger than what is founc. with models of the geomagnetse rield.

Inspection of the coefficients revealed that the error in the derived models indeed contained relatively arse contributions from the Backus sequences. For this purpose the differences $\Delta \mathrm{g}_{\mathrm{n}}^{\mathrm{m}}$ and $\Delta \mathrm{h}_{\mathrm{n}}^{\mathrm{m}}$ between the harmonic coefficients of the model potentlal and those of the potential used as input were tabulated. The coefficients sequences

$$
E_{m}^{m}, 8_{m+2}^{m}, E_{m+4}^{m} \quad \cdots
$$

and

$$
h_{m}^{m}, h_{m+2}^{m}, h_{n+4}^{m}
$$

corresponding to terms which participate in a single Backus series alm invariakly showed the characteristic alternation of signs between cousecutive t.sme which is typical of any such series. Furthermore, when the ratio $R$ of any such term to the leading term of its breries is compared to the correpponding ration $R_{b}$ for the Backus series (Table 4 ), the two ratios are roughly similar.

Re?ults from the test for some of these sequences, a.s well as for some terms not belonging to any sequence, are shown in Table 2 . As can be scen, some of the sequences grow to relatively large amplitudes ( $\mathrm{c} . \mathrm{g}$. the $\mathrm{g}_{2}^{2}$ series for 99 term recovery, the $\mathrm{B}_{3}^{3}$ series for 80 term recovery), others (n.g. the one headed by $h_{2}^{2}$ in the 99 term case) fajl to develop. 
POS IBIE REMEDIES

The counterexamples discovered by Backus satisfy equation (4) only on a single spherical surface. If observations are distributed over a finite region in three dimensions, the series (6) no longer satisfy (4) over the entire region of observation and it is thus possible, in principle, to overcome the problem described earlier. In fact Backus proved [Backus, 1974 ] that equation, (14) cannot be satisfled over a sphericai shell of any finite thickness.

In practical observations such a three dimensionality always cxists due to the finite eccentricity of the orblt of the observing satallite. For those satellites of the $O G O$ serles which were used for geomegnetic surveys this eccentricity was rather small: the altitude range (above sea level) was about 400 to $1500 \mathrm{~km}$ for $0 G 02,400$ to $900 \mathrm{~km}$ for $0 G 04$ and 400 to $1100 \mathrm{~km}$ for $0 G 06$. Unfor'unately, it appears that in the presence of finite error such a varlation in alt:tude is snly able to reduce sor:swhat the effects described in this work and will not eliminate them altogether.

Suppose observations are crifinea to a spherical shell with

$$
r_{0}-\delta r \leq r \leq r_{0}+\delta r
$$

If $\delta r / r=O(\varepsilon)$, then equation $(y)$ - which is the one relevant here - may still bo used, for by Taylor expansion

$$
\begin{aligned}
& u(r, \theta, \varphi)=u\left(r_{0}, \theta, \varphi\right)+o(\varepsilon) \\
& v(r, \theta, r)=v\left(r_{0}, \theta, \varphi\right)+o(\varepsilon)
\end{aligned}
$$

If $u$ and $v$ satisty (9) on the sphere $r=I_{0}$, then the above equations show that they satisfy a $r$ aliar equation over the entire spherical shell, although the "smallness" of the righi iand slac is 
somewhat irpaired. Thes squations (1?) and $(19$ : $:$ ill hold end errur enhancement still exists, 2i+hough nct as nrongly as in the case of observations in an instritely thir sphericai shoz.

As a test, the 99-te: recovery of the 120-tarm model, usec ir. tebles 1 and 2 , was repeated with cata points oscilleting crser a finite range in radial distance, comparable to that of the OGO orbits. A distinet improvemer: in the anfular resolution was obtained (manle 3 ) but there still exists a pronounced discrepancy between $\delta F / F$ and $\delta \lambda$ ! the values of $\delta F / F$ deteriorate sonewhat with increasing $\delta r$, probably becalive the "error" is an unresolved set or $\mathbf{n}=10$ terms, the contribution of which ircreases rapidiy for those points at which $r$ is diminished). If an analysis similar to that of table 2 is performed, it is found that the Backus series are still erhanced, although the ratios $\mathbf{R}$ depart Further from those in table 4 .

More generally, the addition of some vestor data to the data set can in general help resolve the problem. In such cases it might be useful ts separate the model potential $\gamma_{m}$ lerived from $F$ into two parts $\gamma_{m b}$ and $\gamma_{m i}$, in a similar way to the resolution of $\gamma_{e}$ in (16) into its two purts $\gamma_{\mathrm{eb}}$ and $\gamma_{\mathrm{ei}}$ in (17). One may then assime $\gamma_{\text {mi }}$ to be accurately cierived from $F$ end use the added data for deriving $\gamma_{\text {mb }}$ a'.one.

Suppose for example thet one is given as added vector data an accurate location of the dip equator on the surface $r=a$. On the dip equator $\partial r / \partial r=0$ and therefore, by (6) (noting the equality of the two sumations whe: $(7)$ is assumed)

$$
\partial i_{m j} /\left.\partial r\right|_{r=a}=\sum_{m=1}\left(a_{m} \cos m \varphi+b_{m} \sin m \varphi\right) \sum_{n=m}(n+2) G_{n}^{m} p_{n}^{m}(\theta)
$$

Here the left hand side is known, the coefficients $G_{n}^{m}$ are given in table 4 and the unknowns to be derived are the factors $a_{\mathrm{m}}$ and $b_{\mathrm{m}}$. If the variation of $\theta$ is neglected iassuming it to be near $\pi / 2$ ) the solution of (20) reduces to the expansion of a given function of $\varphi$ in a Fourier serjes. In practice, with $\theta$ kept as varlable, the simplest way of deriving $a_{m}$ and $b_{m}$ would be $b_{y}$ least squares Iltting of $(20)$. 
AFPERDDX I : THE BACKUS COUTESEXANDE .

The published form of the counterexample of sackus [ Backus, I910] normalizes the Iegendre functions $P_{n}^{m}$ in the manner preferred by mathematicians and expresses the depenisnce on the longitude angle $\varphi$ in terms of complex exponentials. For computer programs it is much more scruckicnt ta ednnt Gaussian normalization, which avoids the need to calculate square roots when deriving either spherical harmonics or recursion coerificients. Ii is also more corventional in geomagnetic usage to express the dependence on $\varphi$ by means of $\cos m \varphi$ and $\sin m \varphi$ (as in equation 2 ) instead of $\exp ( \pm i m \varphi$ ). The purpose of this appendix is to list some properties of the Becisus counterexamples when these are expressed in Gaussian normalization with trigonometric coefriclents in $\varphi$.

The general form of the counterexample is given in equation $(6)$, and if condition (7) holds, then the two series there are icentical i.e. $G_{n}^{m}=H_{n}^{m}$. The basic recursion relation for either of these series is, with Gaussian normalization,

$$
G_{m+2 k}^{m}=-\frac{(m+2 k)(2 m+4 k+1)(2 m+4 k-1)}{12 k(m+2 k+1)(m+k)} G_{m+2 k-2}^{m}
$$

A similar recursion holds for $\mathrm{H}_{\mathrm{n}}^{\mathrm{m}}$; if (7) is assumed, the results of this recursion are shown in Table 4 . Note that in all terms involved here the sum of indices is even; terms for which this sum is odd vanish icentically.

Table 4 shows that for any given $m$, the magnitude of the coefficients increases with increasing lower index $n$. The rasulting series nevertheles., do converge (ns was shown by Backus), because the normalization factors entering $F_{n}^{m}$ also contribute to the convergence. For Gaussian normalization the $F_{n}^{m}$ for any given $m$ are most conveniently generated by a renursion reletion starting from 


$$
\begin{aligned}
& F_{m}^{m}(\theta)=\sin ^{m} \theta \quad(A-I-2) \\
& P_{m+1}^{m}(\theta)=\cos \theta \sin ^{m} \theta \quad(A-I-3)
\end{aligned}
$$

The relation is

$$
P_{n+1}^{m}(\theta)=\cos \theta P_{n}^{m}(\theta)+\lambda_{n}^{m} P_{n-1}^{m}(\theta) \quad(A-I-4)
$$

where

$$
\lambda_{m}^{n}=(n+m)(n-m) /[(2 n+1)(2 n-1)] \quad(A-I-5)
$$

The derivatives are obtained by recursfors found tron the above ones by difierentiation, starting with

$$
a p_{m}^{m} / d \theta=m \cos \theta \sin ^{m-1} \theta
$$




\section{APPENDIX II : CASE IN WHICH IIERATION FAIIS.}

it was noted that in practice the iteration (13) tends to converge rapialy to an "optimal model" of the lield: the purpose of this apfondix is to point out a case in which this is not true. The exemple given is closely related to the work of Backus [1970] and is probably rather atyfical, but it does show that (13) is not universally useful.

Let $\phi_{2}^{\infty}$ and $\phi_{2}^{\infty}$ be two potentials of the Backus type, satisfying on $r=1$ the condition

$$
\left(\nabla \dot{\varphi}_{1}^{\infty}\right)^{2}=\left(\nabla \phi_{z}^{\infty}\right)^{2}=\frac{2}{F} \quad(A-I I-1)
$$

Iet $\phi_{2}^{n}$ and $\phi_{2}^{n}$ be truncated versions of these series, with all terms having uppe: indices exceeding $n$ anitted, and let

$$
\begin{aligned}
& \left|\nabla \phi_{2}^{n}\right|=\frac{F_{1}^{n}}{2} \\
& \left|\nabla \phi_{2}^{n}\right|=\frac{n}{2}
\end{aligned}
$$

By the theorem of Backus proving the uniqueness of the relation between $\gamma$ and $F$ for potentials with a finite number of harmonic terms [Backus, 1968], $F_{2}^{n}$ unlquely characterizes $\phi_{2}^{n}$ and $F_{2}^{n}$ uniquely characterizes $\phi_{2}^{n}$. However, as $n \rightarrow \infty$, both $F_{2}^{n}$ and $F_{2}^{n}$ tend to $F$ and therefore, for large values of $n$, the difference between the two becomes rather small. 
This suggests that if the input to the recursion (13) consisted of $F_{2}$ or $\frac{F_{2}^{n}}{n}$, with $n$ large enough, the recursive iteration might experience difficulty in telling the two apurt - e.g., given $F_{1}^{n}$, it might start converging to either $\phi_{2}^{n}$ or $\phi_{2}^{n}$, depending on the initial choice of $\gamma_{0}$.

This is confirmed by computer simulations with $F$ generated by 48-term expansions and the least-squares iteration capable of resolving an equal number of coefricients. If the program deriving the model of $\gamma$ is presented by such an $F_{1}^{n}$, one of two things is found to occur. If the initial approximation resembles $\phi_{2}^{n}$ more than it resembles $\phi_{2}^{n}$, the recursive iteration will rapidly converge to the correct coefficlents. If, however, the initial approximation is nearer to $\phi_{2}^{n}$, the coefficients will tend to evolve towards those of $\phi_{2}^{n}$. The Iteration then never converges - the search being conducted in the wrong range of parameters - and Instead the values of the output coefficients wander within a fintte range around those of $\phi_{2}^{n}$.

For a recursion deriving 48 term expansions, each resulting $\gamma$ my be viewed as defining a point in a 48-dimensional space $\sigma_{48}$ in which ea.t coefficient corresponds to one coordinate, and (13) may be v:eweid as prescribing a mapping of each point in $\sigma_{48}$ into another point there. If the field magnitude used as input is $p_{2}^{n}(n \leqslant 6)$, the mapping will have a flxed point $\phi_{1}^{n}$ which maps onto 1tself, and in some region surrounding this point the mapping will converge to $\alpha_{2}^{n}$. However, this convergence evidently does not hold for all of 
$\sigma_{48}$, since for some initial points the process leads to the neighborhool of the "false solution" $\phi_{2}^{n}$. The region from wh1ch the 1teration converges to $\phi_{2}^{n}$ thus has a boundary, which will represent a limit cycle-1.e. If the initial point is located on that boundary, the mapping causes the resulting set of coefficlents to wander along the boundary without either converging to $\phi_{1}^{n}$ or approaching $\phi_{2}^{n}$.

In one simulation the ${ }_{\Lambda}^{\text {coefficients of }} \phi_{1}^{n}$ were

$$
\begin{array}{ll}
B_{1}^{0}=10 & B_{2}^{2}=2 \\
B_{1}^{2}=-2.8 & B_{B}^{2}=3.375
\end{array}
$$

(for the corresponding $\phi_{2}^{n}$, the signs of the $m=2$ terms are reversed). The coefficients of the initial field were chosen as

$$
\begin{array}{ll}
g_{1}^{0}=20 & B_{2}^{2}=2 \\
g_{2}^{2}=-2 \lambda & B_{4}^{2}=2.8 \lambda
\end{array}
$$

For $\lambda=1$ the last two coefficients of the inftial fleld $\Lambda$ equal to coefficients of the "false solutic.n" $\phi_{2}^{n}$ and the recursion in that case heads for that solution (the spurious term $g_{2}^{l}$ is whittled down In the process). On the other hand, if $\lambda=0$ the recursion heads for the "true" solution $\phi_{1}^{n}$. The transition, corresponding to a limit cycle, occurs near $\lambda=0.10$ and when that value was used, as many as 6 1terations were completed before a clear trend became evident. 
REFERENCES

Backus, G.E., Application of a Non-IInear Boundary Value Problem for Laplace's Equation to Gravity and Geomagnetic Intensity Surveys, Quart. J. Mech. Appl. Math. 21, 19, 1968

Backus, G.E., Non-Uniqueness of the External Geomagnetic Field Determined by Surface Intensity Measurements, J. Geophys. Res. I5, 6339, 1970 .

Backus, G.E., Determination of the External Geomignetic Field fram Intensity Measurements, to be published, 1974.

Cain, J.C., I.R. Shapiro, J.D. Stolarik and J.P. Heppner, Vanguard 3 Magnetic Field Observations, J. Geophys. Res. 67, 5055, 1962 •

Cain, J.C., S.J. Hendricks, R.A. Langel and W.V.Hudson, A Proposed Model for the International Ceomagnetic Reference Field 1965, J. of Geomagnetisn and Geolectricity 19, 335, 1967 .

Cain, J.C. and R.E. Sweeney, Magretic Fleld Mapping of the Inner Marnetosphere, J. Geophys. Res. I. 4360, 1970

Cain, J.C., Geomagnetic Models from Satellite Surveys, Rev. Geophys. Space Sct. 2, 259, 1971 .

Cohen, R., Geomagnetic Fleld Inclination Determined by Calibrating the Faraday Rotation of an Incoherently Scattered Signal, J. Geophys.Res. 76, 2487, 1971 .

Heppner, J.P., The World Magnetic Survey, Space Sclence Reviews 2, 315, 1963 •

Woodman, R.F., Inclination of the Geomagnetic Fieid Measured by an Incoherent Scatter Tnchnique, J. Geophys. Res. 76, 178, 1971 •

Zmuda, A.J., A Method for Analyzing Values of the Scalar Magnetic Intensity, J. Geophys. Res. 63, 477, 1958 . 
CAPTIONS TO TABLES

Table 1 Deviations of a model geomagnetic fleld, derived by means of $F$, from the "true" field it is supposed to represent, in a computer simulation. The input rield is given by a scalar potential with 120 coefficients $\left(n_{\max }=10\right)$, the number of points is 1440 and 2 iterations are used, except for one case where the results of 3 iterations are also shown for comparison. All results are in units of $10^{-4}$.

Table 2 Some of the differences $\Delta B=g$ (model) $-g$ (true), where $g$ stands for one of the harmonta coefficients $\delta_{n}^{m}$ or $h_{n}^{m}$ in a simulated recovery of $\gamma$ from $F, j /$ described in Table 1 . Here $R_{b}$ denotes the ratio between a term in a Backus sequence having the type and Indices indicated on the table and the leading term of its sequence (these iatios are lifs ind in Table 4), while $R$ is the ratio between corresponding values of $\Delta_{B}$.

Table 3 Results similar to those of Table 1 , with a similar input model and 99-term recovery in 3 iterations, for cases in which data points are spread out over a spherical shell bounded by $r_{0} \pm \delta x$.

Table 4 Ratios $G_{m}^{m}+2 k / G_{m}^{m}$ derived from the recursion relation A-I-1. 


\begin{tabular}{|l|c|c|c|c|}
\hline No. of terms & 63 & 80 & \multicolumn{2}{|c|}{99} \\
\cline { 4 - 5 } in recovery & & & 2 1ter. & 3 1ter. \\
Niean $\delta \mathrm{F} / \mathrm{F}$ & 26 & 20 & 1.1 & 6.4 \\
Weanst $\delta \mathrm{F} / \mathrm{F}$ & 249 & 207 & 35.0 & 31.4 \\
Worst $\delta \lambda$ (radians) & 2497 & 2139 & 1298 & 106.4 \\
\hline
\end{tabular}

Tarle 1 


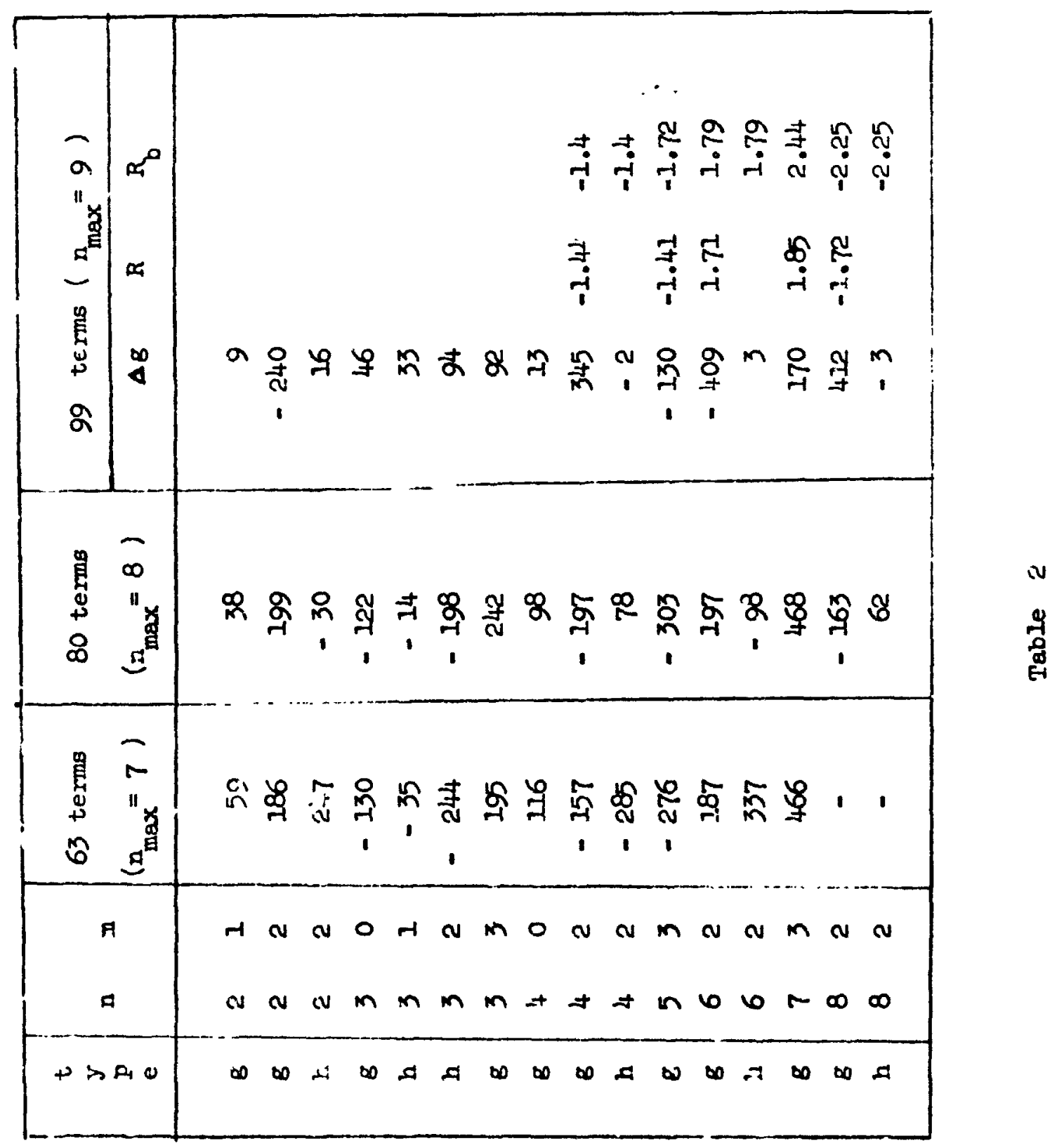




\begin{tabular}{|c|ccc|}
\hline$\delta r / r_{0}$ & $0 .$. & $0 . C 5$ & 0.10 \\
\hline Mean $\delta F / F$ & 6.37 & 7.21 & 9.17 \\
Worst $\delta F / F$ & 31.4 & 79.9 & 135.2 \\
Mean $\delta \lambda$ & 106.4 & 85.6 & 79.7 \\
Worst $\delta \lambda$ & 1307 & 1303 & 1050 \\
\hline
\end{tabular}

Table 3 


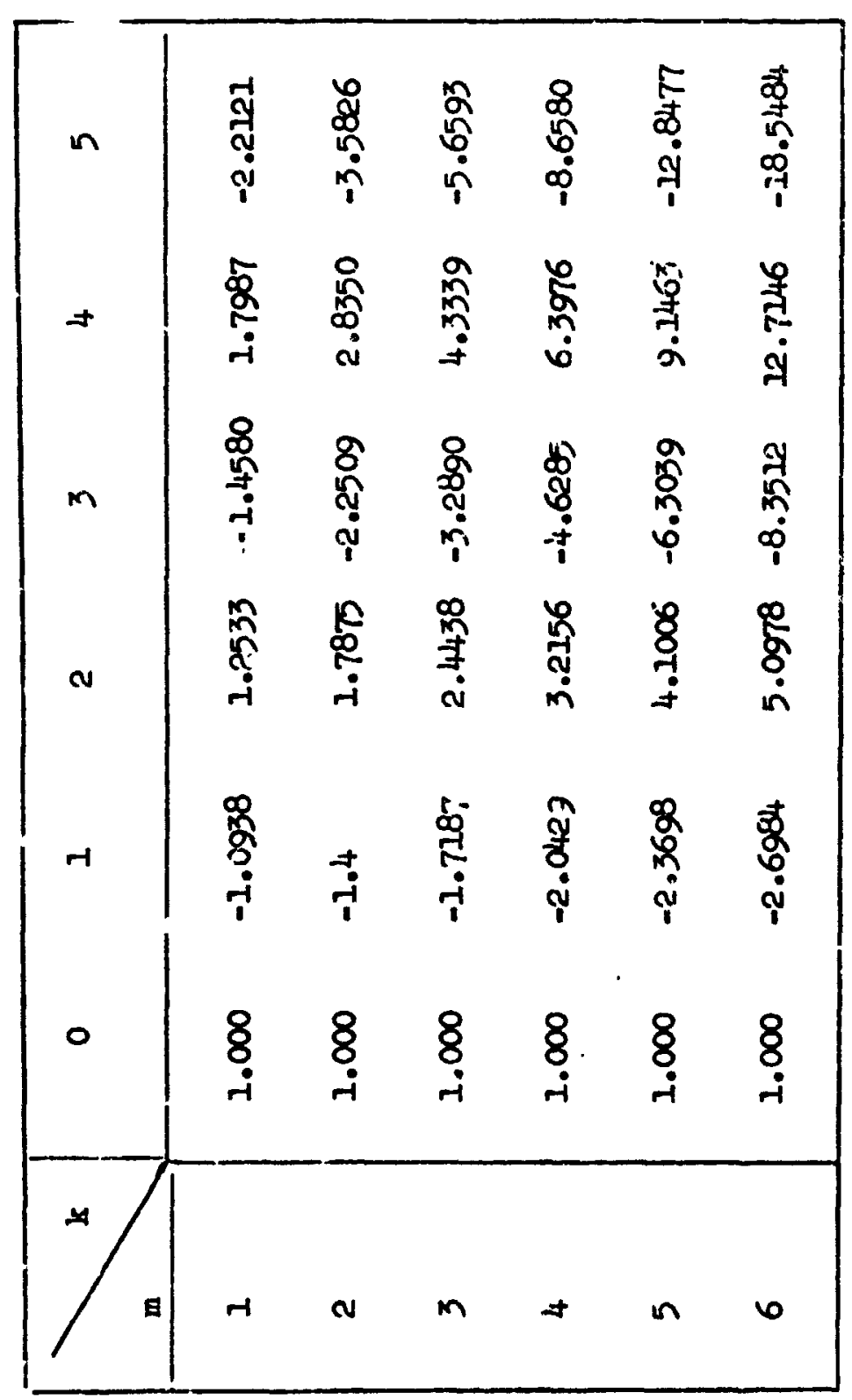

